\title{
MCCM: Multilevel Congestion Avoidance and Control Mechanism for Mobile Ad Hoc Networks
}

\author{
Md. Manowarul Islam \\ Dept. of Information and Communication Technology, Mawlana Bhashani Science and Technology University, Tangail, \\ Bangladesh \\ Email: manowar_cse_du@yahoo.com
}

Md. Abdur Razzaque

Dept. of Computer Science and Engineering, University of Dhaka, Bangladesh

Email: m_a_razzaque@yahoo.com

\author{
Md. Ashraf Uddin \\ Dept. of Computer Science and Engineering, Mawlana Bhashani Science and Technology University, Tangail, \\ Bangladesh \\ Email:ashraf_16csedu@yahoo.com
}

\begin{abstract}
A.K.M Kamrul Islam
Dept. of Computer Science and Engineering, Mawlana Bhashani Science and Technology University, Tangail, Bangladesh

Email: kamrul3000@gmail.com
\end{abstract}

\begin{abstract}
Congestion in Mobile Ad Hoc Network causes packet loss, longer end-to-end data delivery delay which affects the overall performance of the network significantly. To ensure high throughput, the routing protocol should be congestion adaptive and should be capable of handling the congestion. In this research work, we propose a Multilevel Congestion avoidance and Control Mechanism (MCCM) that exploits both congestion avoidance and control mechanism to handle the congestion problem in an effective and efficient way. MCCM is capable of finding an energy efficient path during route discovery process, provide longer lifetime of any developed route. The efficient admission control and selective data packet delivery mechanism of MCCM jointly overcome the congestion problem at any node and thus, MCCM improves the network performance in term of packet delivery ratio, lower data delivery delay and high throughput. The result of performance evaluation section shows that, MCCM outperforms the existing routing protocols carried out in Network Simulator-2(NS-2).
\end{abstract}

Index Terms - Mobile Ad Hoc Network, Energy Efficient Routing, Path Usability, Congestion Control, Admission Control, Selective Packet Delivery

\section{INTRODUCTION}

Mobile Ad Hoc Network (MANET) [1] is an infrastructure-less network where a number of mobile nodes can communicate with each other by developing a multi-hop route. The nodes are self-organizing, selfconfigurable and can organize themselves arbitrarily [2]. Nodes in such a network contend for bandwidth during data transmission. So routing protocol should provide an efficient way to improve the performance of the network by ensuring maximize use of network resource like battery energy and bandwidth, etc. An energy efficient routing methodology can improve network performance and provide longer lifetime of each node in MANET. Due to the dynamic behavior and limited transmission range, the lifetime of an established route is very short. All the mobile nodes in a MANET network are operated on their limited battery energy [3] [4] [5]. Lifetime of a mobile node depends on their limited battery energy. So, designing and developing an efficient routing methodology is a challenging problem.

Congestion in Mobile Ad Hoc Network leads packet loss, queue overflow and low throughput in MANET [6] [7] [8]. For a congested network, nodes in active route cannot deliver the data packets effectively and thus a lot of packets are dropped. Thus, retransmitting process of these dropping packets introduces higher end-to-end data transmission delay. Congestion consumes nodal residual battery energy unnecessarily. To mitigate the congestion, nodes have to consume extra energy leading low throughput. The efficient congestion avoidance and control mechanism can achieve high throughput by efficient congestion controlling mechanism. The dynamic behavior, limited energy and transmission range causes the congestion control a subjective research for wireless communications. In this work, we propose a mechanism that detects multilevel congestion status at each node and avoids the congestion efficiently. During the route discovery process, MCCM finds a route from source to destination node that ensures maximum use of limited energy of mobile nodes. This energy efficient path provides highest longevity, saves the energy and ensures high performance of the network. MCCM also provides admission control mechanism that controls the admission 
of various control messages to make the network congestion free and its selective data delivery mechanism mitigates the congestion in an efficient way.

Nodes in a MANET contend for the shared medium for data transmission. So congestion may occur as the link between the nodes breaks due to node mobility, low battery power, media overloading, etc. Since nodes in MANET network can act as both a host and a router, they have to exchange data packets from multiple senders or receiver at the same time. So a queue overflow or channel or media overloading may occur [9]. As a result, node cannot deliver the data packets efficiently. Thus congestion leads to packet drops, degradation of throughput and increases the network end-to-end data delivery time [6] [7].

Consider Fig. 1, there are three routes to destination node D from three source nodes $\mathrm{S}, \mathrm{X}$ and $\mathrm{E}$, respectively. All the sources $\mathrm{S}, \mathrm{X}$ and $\mathrm{E}$ send data via the intermediate node $\mathrm{B}$. The node $\mathrm{B}$ receives data packets and forwards them to the destination node D. When only one route $S \rightarrow$ $B \rightarrow D$ is active, $\mathrm{B}$ can deliver data packets properly. This is normal situation as queue occupancy at node $B$ is normal. But when all the three nodes $\mathrm{S}, \mathrm{X}$ and $\mathrm{E}$ start delivering data, queue length at node $\mathrm{B}$ increases rapidly. Since the incoming data rate is much higher than the forwarding data rate, it cannot deliver them properly to the destination node. As a result, packets may drop since queue overflow occurs at node B. Thus, the network may suffer from high packets loss, long end to end data delivery delay and low throughput due to congestion.

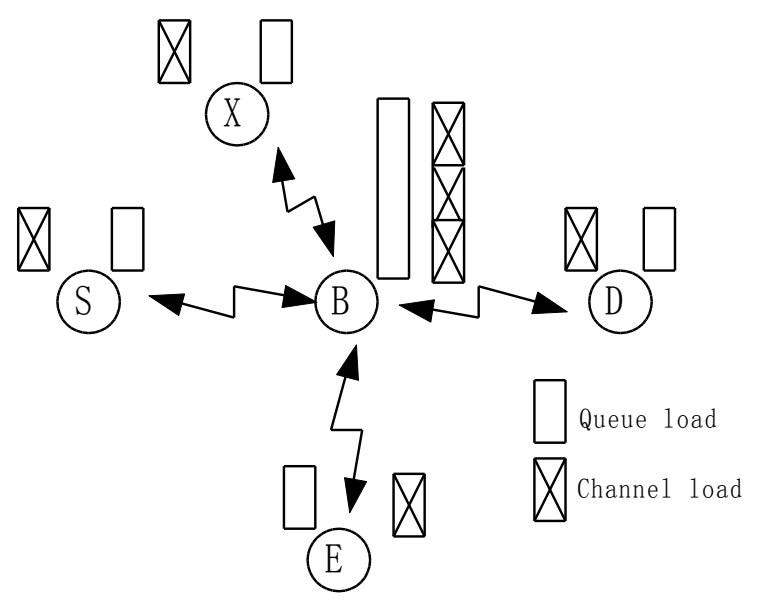

Fig. 1. Congestion occurs in the network due to queue over-flowing or channel overloading

Again, nodes in MANET share the transmission channel. Nodes may contend for the channel for transmitting the data packets leading packet collisions in the network. Again consider Fig. 1, the channel loading at node B is raised to a high level which affects the network throughput significantly. This is because, packets from others nodes may collide at node B since it cannot store the incoming packets properly. Therefore, the node will also be unable to transmit data packets successfully. As a result, queue will overflow at this node and packets start dropping. So the network becomes a congested one since there is huge packets will loss at node B due to the channel overloading. Spilt Multi-path Routing (SMR) [10], was proposed, where nodes use multiple alternative routes to the destinations. These alternative routes are used during the congestion by splitting the traffic load. But, the problem is that multiple route maintenance overhead affects the network throughput.

In this paper, we propose a Multilevel Congestion Avoidance and Control Mechanism (MCCM), in which destination node selects an energy efficient route that is developed based on path usability and ensures the maximum use of network resources (e.g. battery energy, limited bandwidth). Its multilevel congestion detection and avoidance mechanism can detect multiple levels of congestion proactively. MCCM provides an efficient admission control approach to avoid congestion. It also develops a selective packet delivery mechanism during the congestion and provides an efficient way to make the network congestion free by delivering the high priority packets selectively. The main contributions of this work are summarized below:

- The route discovery process of MCCM provides an efficient way to find a path that ensures the maximum use of network resources.

- MCCM provides efficient congestion avoidance and control mechanism, where nodes can detect multilevel congestion and take proper actions to avoid congestion.

- The admission control approach can avoid congestion proactively and thus, increases network throughput.

- For a congested node, its selective packet delivery mechanism mitigates the congestion considerably.

The rest of this paper is organized as follows. We describe related works and motivation in Section 2 and Network model and assumptions in Section 3. Our proposed mechanism MCCM is presented in Section 4 and the simulation results are presented in Section 5. Finally, we conclude the paper in Section 6 along with future research direction.

\section{RELATED WORKS}

In recent years, many congestion control protocols have been proposed to mitigate congestion in MANET. Most of them used the concept of load balancing. They use multiple routes to balance traffic loads on the event of congestion [11] or route failures and thus improve the network performance. For example, a Multi-path DSR protocol (MP-DSR) [12] improves QoS with respect to end-to-end reliability; Spilt Multi-path Routing (SMR) [10] uses multiple paths to split the network traffic and reduces the congestion. Nodes in congestion adaptive Routing Protocol (CARP) [13] maintains both primary and bypass routes; if need, use bypass routes to mitigate congestion, etc. But, the problem is that, multiple route maintenance overhead affects the network throughput. Congestion Aware and Adaptive Dynamic Source 
Routing Algorithm (M-DSR) [14] with Load-balancing in MANETs maintains multi-path routing and searches their primary and secondary caches for multiple routes to the targeted destination.

$\mathrm{E}^{2} \mathrm{AODV}$ is another approach that uses the concept of load balancing for efficient uses of the energy [15]. It uses multiple routes to avoid the congestion in the network. A node in the network will be considered as an eligible node only if it has a minimum amount of energy. Though it has some route to the destination node it cannot deliver data packets if its energy level goes below to this threshold value. Another important problem is the high overhead of maintaining multiple routes.

Early Congestion Detection and Self Cure Routing in MANET (ECDSCAODV) is a technique that reduces the congestion in the network based on queue utilization at each primary route [16]. The node warns its neighbors node about the congestion based on current status. During the congestion, it tries to find alternative route. On finding an alternative route, nodes transmit their dropped packets. In Early detection congestion and control routing in MANET (EDCAODV) is another congestion aware routing protocol where the nodes can detect the congestion in advance [17] and find the best alternative path from a series of alternatives path to the destination and delivers the data packets. Maintaining a number of alternative routes is the main problem since it is required more energy and consumes network resources unnecessarily. Though these two protocols detect the congestion early and find some alternative routes that are congestion free; finding these routes and keeping information all of these routes is costly. Thus they reduce the network performance.

The authors of the early congestion detection and adaptive routing in MANETs (EDAPR) show that, nodes of active route preserves information about some noncongested (NHN) neighbor nodes [18]. During the congested state, node sends a message to these NHN nodes. So these NHN nodes try to find some alternative routes and adapt the data transfer rate and overcome congestion. Though it provides higher performance than EDCAODV and ECDSCAODV, it has some disadvantages. Finding alternative route using noncongestive nodes may need some extra resources, decreases node lifetime. The multiple NHN may try to deliver same data packets. Again, keeping information about all the NHN nodes increase the network overhead.

The aforementioned protocols can handle congestion by load balancing using alternative routes. Some protocols detect the congestion at early stage and mitigate congestion by self-curing methods or use alternative route during congestion or maintain some non-congested nodes. The non-congested nodes discover alternative route and overcome the congestion. The use of multiple routes or nodes reduces network throughput consuming network limited resources. To make the efficient use of network resources we propose MCCM routing protocol that discovers an energy efficient route. Nodes in the network are capable of finding multilevel congestion status and take proper actions. The admission control mechanism avoids the congestion proactively. To mitigate congestion, MCCM provides a selective delivery of data packets based on their priority and usability of the route. Thus, MCCM does not need to maintain extra alternative routes or any non-congested node to handle the congestion.

\section{NETWORK MODEL AND ASSUMPTIONS}

Mobile Ad Hoc Network is a wireless network where the mobile nodes act like forwarder node in infrastructure less environment. Example of such nodes may be laptop, mobile phone, sensor nodes etc. We consider a network where mobile nodes exchange their data packets using multipath routing. In our propose mechanism, a destination node can select a route that is more energy efficient than other routes and each node is capable of detecting the congestion level more effectively and takes proper steps to keep the network congestion free. For the simulation purpose and performance evaluation, we choose AODV as an underlying routing layer protocol; it is an on-demand routing protocol. What follows, we describe the queue management of a mobile node and the internal structure of any intermediate node of the network.

\subsection{Network Layer Queue Management}

In MANET a node delivers data and control packets for multiple source-destination pair nodes. All the data packets are queued and delivered from the network layer queue. So the incoming traffic for a node must be in a rate such that the node can deliver them properly to make the network congestion free. As shown in Fig. 2, node receives data packets from multiple neighbor nodes and stores them in network layer queue before delivering to the next hop of the route.

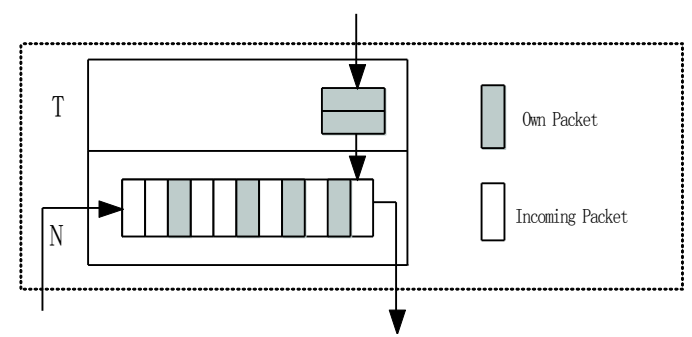

Fig. 2. Network layer queue of any node

Again, the node can creates and delivers data packets for other nodes. To ensure a congestion free network, the total number of packets in the queue at any moment should not exceed the queue length. The packets filled with color in figure 2 are labeled as own generated packets and are inserted into network layer queue from upper layer and the others are received packets from neighboring nodes. So, total queue occupancy of any node depends on two things (i) its own packet generation rate and (ii) total number of receives packets from neighboring nodes. Let $P_{\text {in }}$ is the total number of incoming packets and $P_{\text {gen }}$ is number of generated packets and $Q_{\text {size }}$ is the total queue length of any node, then the node should maintain the following equation to avoid congestion. 


$$
P_{\text {in }}+P_{\text {gen }}<Q_{\text {size }}
$$

The equation implies that, if the incoming data packets exceed the queue size then queue overflow will occur at the node and it will be congested.

\subsection{Internal Structure of a Mobile Node}

The congested node drops data packets since it cannot deliver them properly. So congestion is one of the critical problems that causes low throughput. Congestion avoidance can improve the network performance significantly. It is not possible to build a network which is totally congestion free due to the dynamic topology, limited transmission range and energy constraint of the mobile nodes. In MCCM, a node can detect multilevel congestion and perform several actions to avoid congestion proactively. The internal structure of any node in the network is shown in Fig. 3.

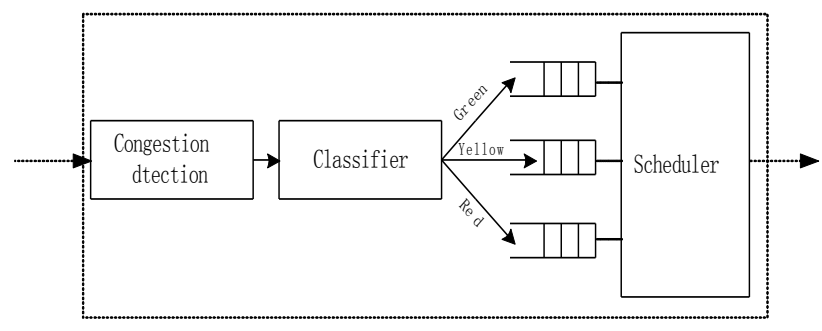

Fig. 3. Internal structure of a mobile node

The congestion detection unit of the node is capable of detecting the congestion level based on the current queue occupancy. For a congested node, the classifier unit can classify the data packets into three categories, namely green, red and yellow based on their priority. The scheduler of the node takes appropriate decision whether to deliver or drop packets by examining the packets priority which will be described in section 4 in details.

\section{THE MCCM ARCHITECTURE}

Congestion in MANET causes a lot of data packets and degrades the network performance significantly. The proposed MCCM is capable of detecting multilevel congestion at node level and control the congestion efficiently. During the route discovery phase, node finds a route that is more energy efficient and provides longer life time. Node can control the admission of packets into the network layer queue so that the network remains at a non-congested state. But if the intermediate node detects congestion due to buffer overflow or limited energy or any other reasons, it tries to mitigate the congestion by efficient packet delivering mechanism. The node assigns priority to the queued packets and delivers the data packets which has highest priority level and drops the others packets for making the network congestion free. What follows, we discuss the route discovery process of MCCM and the multilevel congestion detection and control mechanisms.

\subsection{Route Discovery Mechanism in MCCM}

In MCCM, an energy efficient route is developed based on residual energy at each intermediate node. When a source node initiates a route discovery process by flooding route request (RREQ) messages, it appends its own energy ratio as $E_{\text {res }}^{\text {min }}$ for the expected route, where $E_{\text {res }}^{\min }$ is the ratio of current residual energy to initial energy.

$$
E_{\text {res }}^{\text {min }}=\frac{E_{r e s}^{\text {cur }}}{E_{\text {res }}^{\text {init }}}
$$

After receiving the RREQ message, an intermediate node calculates its current energy ratio and compares it with the receiving $E_{r e s}^{\min }$. It then updates $E_{r e s}^{\min }$, if it is smaller than $E_{r e s}^{\min }$. This process ensures the developing a route with minimum residual energy.

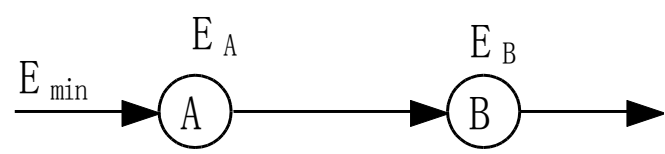

Fig. 4. E $\mathrm{E}_{\text {res }}^{\min }$ calculation between two nodes

Consider an example in figure 4, node A receives the minimum energy $E_{\text {res }}^{\text {min }}$ from its previous node. It then compares its own energy ratio with the received $E_{\text {res }}^{\text {min }}$ and updates $E_{\text {res }}^{\text {min }}$, if it is smaller than $E_{\text {res }}^{\min }$. After appending $E_{\text {res }}^{\text {min }}$ in RREQ message, node A forwards the RREQ message to node B. In this manner, when the destination node gets the first RREQ message, calculate the usability of the path using (3).

$$
P U_{1}=\frac{E_{r e s}^{\min , 1}}{H^{1}}
$$

Here $H^{1}$ is the number of hop between the source and the destination nodes. The destination node waits for a time $T_{k}$ to receive more RREQ messages and calculates the usability of each path $k$ using equation 4 :

$$
P U_{k}=\frac{E_{r e s}^{\min , k}}{H^{k}}, \forall k \in N
$$

Where $\mathrm{N}=1,2,3 \ldots \mathrm{N}$ is the total number of routes from which the destination node get RREQ message, $P U_{k}$ indicates the path usability of route $\mathrm{k}$ and $H^{k}$ is the hop count. The equation 3 implies path usability $P U_{k}$ of any path $k$, depends on the residual energy and the hop count of the route. The higher the residual energy, the better the path usability is. On the other hand, route $k$ is energy efficient with minimum number of hop count $H^{k}$. The destination node will choose a path from the list of path, $P_{\text {sel }}$ that has the higher usability using (5).

$$
\begin{aligned}
P_{\text {sel }} & =\max \left\{\frac{E_{r e s}^{\min , 1}}{H^{1}}, \frac{E_{r e s}^{\min , 2}}{H^{2}}, \ldots \frac{E_{r e s}^{\min , N}}{H^{N}}\right\} \\
& =\max \left\{P U_{1}, P U_{2}, P U_{3} \ldots P U_{N}\right\}
\end{aligned}
$$

After choosing an energy efficient path, the destination node informs the source node by replying a route reply (RREP) message. The source node starts delivery of data 
packets after getting RREP message. Thus, MCCM develops an energy efficient route. The path usability of MCCM ensures a path that is energy efficient, reliable and provides higher lifetime during the data transmission process. Thus, MCCM preserves maximum use of limited resource of mobile nodes.

\subsection{Congestion Control in MCCM}

In MANETs, nodes always contend for the shared medium to transmit data packets to other nodes. If a node cannot forward the packet according to incoming rate, then the node enter into congested state and has to drop a lot of packets. Such a congestive state may cause many problems like longer end-to-end data delivery delay, low throughput and high packet loss etc. To cope up with the above drawbacks, in MCCM node can measure their congestion level based on their current queue length and send a Congestion Status Packet (CSP) to its neighbor nodes to inform about the current congestion status. MCCM divides the node's congestion status into safe (zone-I) and unsafe zone (zone-II) respectively. The zone I and zone II only indicate the non-congested and congested state. But these two zones do not provide enough information whether a node is going to be congested one within a few moments or not. To measure the congestion level more accurately, MCCM further divides these two zones into four states namely lightly loaded, medium loaded, fully loaded and congested state.

\subsubsection{Multilevel Congestion Detection Process}

Nodes in a MCCM network check their own network layer queues to detect the congestion status. We first define thresholds namely $T h_{\text {mid }}$ which indicates the midpoint of the Zone I and Zone II provides basic information whether the node lies in a non-congested or it is going to fall in congested state. If the current queue occupancy at any node is below this threshold, then the node is considered as a non-congested node and it can deliver the data packets properly. This warning threshold $T h_{\text {mid }}$ for any node $i$ is defined as follows:

$$
T h_{\text {mid }}^{i}=\alpha \times Q L_{\text {size }}^{i}
$$

Here, the value of $\alpha$ is $60 \%$. To define a loaded and congested state, another two thresholds $T h_{\min }$ and $T h_{\max }$ are defined by the queue status as follows:

$$
\begin{aligned}
& T h_{\text {min }}^{i}=\beta \times Q L_{\text {size }}^{i} \\
& T h_{\text {mid }}^{i}=\gamma \times Q L_{\text {size }}^{i}
\end{aligned}
$$

Where, $\beta$ and $\gamma$ are two control parameters; in our simulation, we set $\beta=0.40$ and $\gamma=.80$, respectively. The value of $\gamma$ indicates that node $i$ enters into the congested state if the current queue occupancy is greater than $80 \%$ of total queue size.

The average queue occupancy of a node $i$ can be measured periodically using Exponentially Weighted Moving Average (EWMA) formulae as follows:

$$
Q L_{\text {avg }}^{i}=(1-\sigma) Q L_{\text {avg }}^{i}+Q L_{\text {cur }}^{i} \times \sigma
$$

Where $\sigma$ is a weighted factor and $\mathrm{QL}_{\text {cur }}^{\mathrm{i}}$ is the current queue size of node $i$. Now, based on the value of queue

\begin{tabular}{|c|c|}
\hline Congestion Level & Queue status \\
\hline Lightly Loaded & $Q L_{\text {avg }}^{i} \leq T h_{\text {min }}^{i}$ \\
\hline Medium Loaded & $\begin{array}{c}Q L_{\text {avg }}^{i}>T h_{\text {min }}^{i} \\
\text { but } \\
Q L_{\text {avg }}^{i} \leq T h_{\text {mid }}^{i}\end{array}$ \\
\hline Fully Loaded & $\begin{array}{c}Q L_{\text {avg }}^{i}>T h_{\text {mid }}^{i} \\
\quad \text { but } \\
Q L_{\text {avg }}^{i} \leq T h_{\text {max }}^{i}\end{array}$ \\
\hline Congested & $Q L_{\text {avg }}^{i}>T h_{\text {max }}^{i}$ \\
\hline
\end{tabular}
occupancyQ $\mathrm{L}_{\text {avg }}^{\mathrm{i}}$, node $i$ determine the congestion status as table-1:

Table 1. Congestion Status based on queue occupancy

\subsubsection{Congestion Handling Mechanism}

After detecting the own congestion status nodes inform their neighbor nodes by exchanging a periodic message called Congestion Status Packet (CSP). Nodes try to avoid congestion proactively and reduce the chance of entering into the congested zone. For avoiding the congestion, any node $i$ in MCCM can take one of the following steps according to their congestion level:

- Normal Operation: If current congestion status of node is lightly loaded then it acts like a forwarder node and performs it normal operation and forwards the data and control packets to the next hop using the energy efficient path developed earlier.

- Admission Control: The efficient admission control mechanism of MCCM keeps the node congestion free to make the network more useful. During the medium loaded and loaded states MCCM control the admission of both data and control packets through any node to overcome the congestion problems effectively.

- Selective packet delivery: If any node falls into congested state due to queue overflow or packet collision, the selective packet delivery approach of MCCM selectively delivers some data packets from its queue based on the packet priority until the network become normal and hence make the network congestion free. What follows we describe the admission control and selective data delivery mechanisms of MCCM.

\subsubsection{Admission Control Mechanism}

In order to make the network congestion free, MCCM provides an efficient admission control mechanism. In case of medium loaded status, approximately $60 \%$ of the total queue length is loaded. Since broadcasting process for RREQ message is highly redundant and flooding of RREQ messages will fill the queue more quickly. Again, broadcasting of RREQ means allowed the node to create some routes through the node and more data packets will 
exchange using the node. Thus the node will be a congested one within a short period of time. To overcome this problem, MCCM will drop all new RREQ messages from the network layer queue shown in Fig. 5(a) and the node can deliver the data packets for the existing routes in a frequent manner. Thus, MCCM reduce the chance of congestion during medium loaded situation.

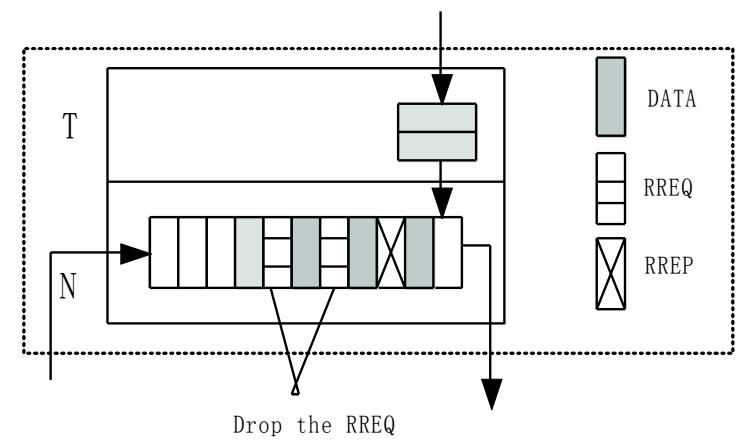

(a) Admission Control of RREQ

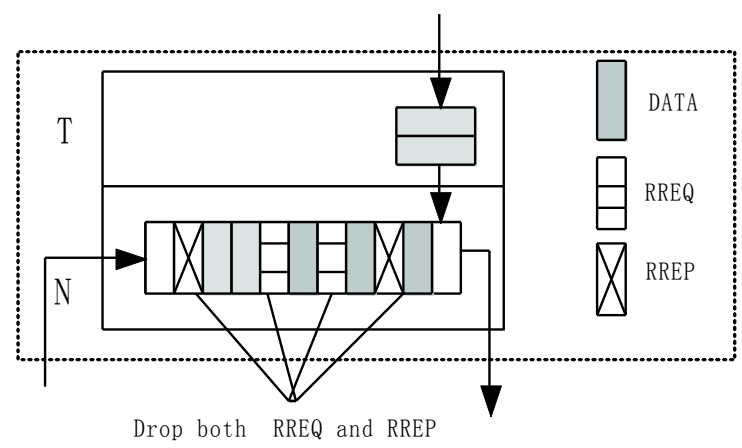

(b) Admission Control of RREQ and RREP

Fig. 5. Admission control during medium loaded and loaded condition

During the fully loaded state, a silly increasing of packet generating rate at each source causes the receiving forwarder node to enter onto congested mode since its queue occupancy during loaded state is about $80 \%$. In such a case node allows only to deliver the data packets. The new RREQ and RREP packet will discard to ensure that the node remains in a congestion free state. This process is shown in Fig. 5(b). In addition, the node will create an alarming message namely Congestion Notification message (CNM) and send it to all the source nodes to inform the current congestion status. After receiving the CNM, all the participating source nodes will not increase their delivery rate until the network become normal. Thus, MCCM control the admission of the packets and overcome the congestion of the network proactively.

\subsubsection{Selective Packet Delivery Mechanism}

In this section, we will describe the packet delivery and dropping methodology of a node during the congested state. When the average queue length at any node exceeds the maximum threshold level, the node becomes congested. To control the congestion, MCCM assigns priority to the queued packets. This is done by considering traversed node of the total route and path usability of the route.

If a packet traversed more than it half way, it will be given higher priority to delivery than a packet that traversed less number of nodes of the route. Table 2 shows the priority level of queued packet base on their route traversing status.

Table 2. Packets priority during congestion

\begin{tabular}{|c|c|}
\hline Number of node traversed & Priority, $\boldsymbol{P}_{\text {level }}$ \\
\hline Below $\frac{1}{2} \times H_{c}$ & .25 \\
\hline Above $\frac{1}{2} \times H_{c}$ & .75 \\
and \\
Below $\quad \frac{3}{4} \times H_{c}$ & \\
\hline$\frac{3}{4} \times H_{c}$ or above & 1 \\
\hline
\end{tabular}

The route that has higher residual energy will also achieve higher priority to deliver data packets. For every packet $P_{j}$ where $j=1,2,3 \ldots j$ at node $i$ in the network, we define its priority as follow:

$$
\begin{aligned}
P R_{j}^{i} & =P U_{k} \times P_{\text {Level }}^{j} \\
& =\frac{E_{\text {res }}^{\text {men }}, k}{H^{k}} \times P_{\text {Level }}^{j}
\end{aligned}
$$

Table 2 shows that, if a packet visited more than $70 \%$ of the path then it will achieve higher priority and considered as a deliverable packet. Equation 10 implies that if a route has higher residual energy or the higher path usability, packets of that route will achieve the higher priority for delivery and MCCM ensure the energy efficient routing. Packets of a route having lower residual energy and resides closely to the source node will assign lower priority and these packets will be dropped to mitigate the congestion. Thus, a node can easily calculate the priority of each packet. It then categorizes the packets by the classifier unit into three levels as green, yellow or red based on their priority. The lowest $30 \%$ will fall into red category, $40 \%$ will in yellow category and highest $30 \%$ into green. Finally it takes appropriate actions as follow:

- GREEN: The first $30 \%$ packets are considered as GREEN having higher priority. This type of packets will be considered as deliverable packets since they are nearer to the destination node and corresponding route has highest residual energy.

- YELLOW: The second 40\% packets are considered as YELLOW and the node will not deliver the packets until the network becomes normal. The data packets will be queued for a certain period of time $T_{\text {wait }}$ If the node becomes normal within $T_{\text {wait }}$, then it will start delivery of these waited packets.

- RED: The third 30\% packets are considered as RED. Packets those are closely to the source region and having lower usability will fall in this category. In such a case, node will drop these packets from the queue to reduce the congestion. 
The algorithm 1 given below, shows the operations of a congested node $i \in N$ of an active route.

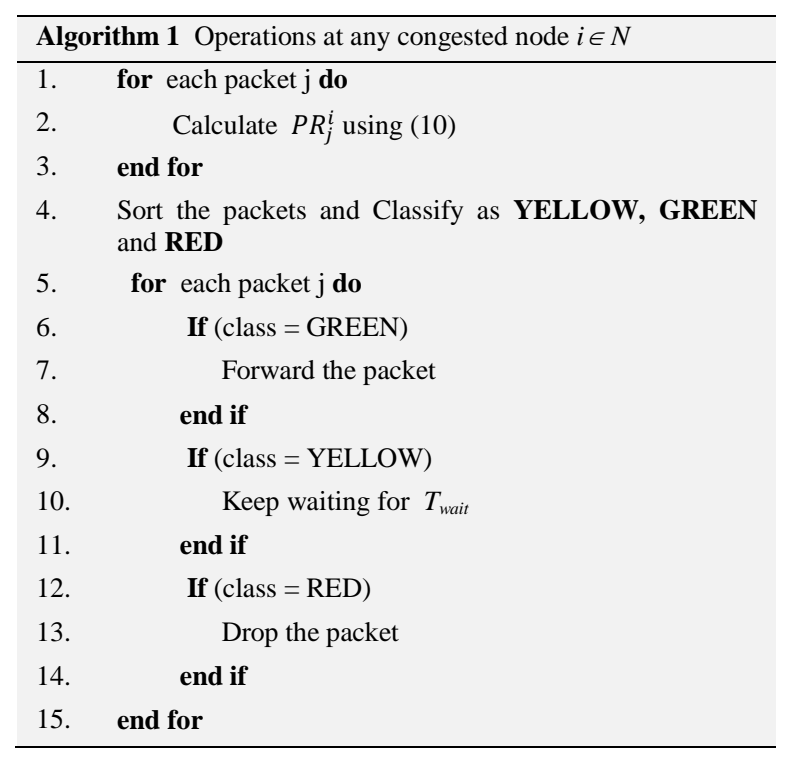

\section{PERFORMANCE EVAlUATION}

We evaluate the performance of our proposed MCCM mechanism in network simulator v-2.34 [19]. We compare the simulation results with other two existing protocols that are closely related to our works namely SMR [10], EDAPR [18]. The results of our simulation state that the MCCM outperforms the other protocols.

\subsection{Simulation Environment}

We consider a square area of size $1200 \times 1200 \mathrm{~m}^{2}$, where 100 mobile nodes are deployed randomly. The simulation time is set to 250 seconds. The transmission range of the nodes is $250 \mathrm{~m}$. To vary the traffic at each node, we vary the packet generation rate at each source node from 1 to 8 packets per second and the size of data packet is 512 bytes and the underlying transport and MAC layer protocols are UDP and IEEE 802.11 DCF, respectively. The table 3 summarizes the overall simulation parameters that we use for simulation purpose.

Table 3. Simulation Parameters

\begin{tabular}{|l|l|}
\hline \multicolumn{1}{|c|}{ Parameter } & \multicolumn{1}{c|}{ Value } \\
\hline Network area & $1200 \mathrm{~m}$ x $1200 \mathrm{~m}$ \\
\hline Number of nodes & 100 \\
\hline Deployment type & Random \\
\hline Number of sources & 25 \\
\hline Node movement model & Random waypoint \\
\hline Transmission range & $250 \mathrm{~m}$ \\
\hline Transport layer protocol & UDP \\
\hline MAC layer protocol & IEEE $802.11 \mathrm{DCF}$ \\
\hline Bandwidth & $11 \mathrm{Mbps}$ \\
\hline Data packet size & 512 bytes \\
\hline Data packet generation rate & 1 to 8 packets/sec \\
\hline Propagation model & Free Space \\
\hline Weight factor $\sigma$ & 0.002 \\
\hline
\end{tabular}

\subsection{Performance Metrics}

We use three performance metrics those are as follows:

- Packet delivery ratio: The packet delivery ratio is measured as the ratio of the total number of received data packets by all the destination nodes to the total number of generated data packets.

- Average end-to-end packet delay: It is measured as the average time (in $\mathrm{ms}$ ) required by a data packets to reach from source to the destination node.

- Normalized routing overhead: For each successfully delivered data packet the number of control packets generated during the simulation period considered as normalized routing overhead.

\subsection{Impact of varying traffic loads}

To evaluate propose MCCM, we first study the impact of varying the packet generation rate at each source node. We continuously increase the packet generation rate from $1 \sim 8$ packets per seconds, i.e., from $0.5 \sim 4.0 \mathrm{KBps}$ at each source. We keep the nodes mobility constant value at 2 $\mathrm{m} / \mathrm{s}$ within the network. The graphs in Fig. 6 compare the performances of the studied protocols with MCCM.

The packet delivery ratio (PDR) for all the protocols decreases sharply with increasing packet generation rate, as shown in Fig. 6(a). This is because, each intermediate node cannot deliver the data packets with the receiving rate i.e., the forwarder nodes cannot deliver as many packets as they receive. The graph also shows that, our proposed MCCM mechanism outperforms their protocols.

SMR balance the traffic using multiple routs. With the increasing traffic rate, congestion may occur at multiple routes leading packets loss and thus provides lower packet delivery ratio. EDPAR maintains multiple NHN (non-congested) nodes during congestion and finds alternative routes. It performs better than SMR, but provides lower packet delivery rate than MCCM. MCCM can measure the normal and congestive states more accurately and can take appropriate control actions to handle the congestion. Besides its energy efficient route provides longer lifetime which ensure reliable data delivery. The selective packet delivery mechanism of MCCM reduces the packet dropping rate significantly. As a result, it can deliver data packets much higher than SMR and EDAPR.

Fig. 6(b) shows the average end-to-end packet delivery delay of the protocols for various traffic loads. As expected theoretically, the end-to-end packet delivery delay increases with the packet generation rate. Congestion may occur as the network traffic increases over time. As a result, nodes in SMR experience highest level of congestion among the multiple route which causes highest end-to-end delay. A lot of packets dropping increase the delay. In EDAPR node can detects the congestion level and using the NHN find an alternative route. Multiple NHN nodes may contend for the medium and thus increases the delay on finding the alternative routes. MCCM's multiple congestion avoidance and control mechanism handles congestion proactively. It does not allow an intermediate node to increase the traffic 
by creating new connections if the node is in Medium Loaded state and thus control the network traffic. As a result, MCCM reduces the packet dropping rate and provides lowest delay than SMR and EDAPR.

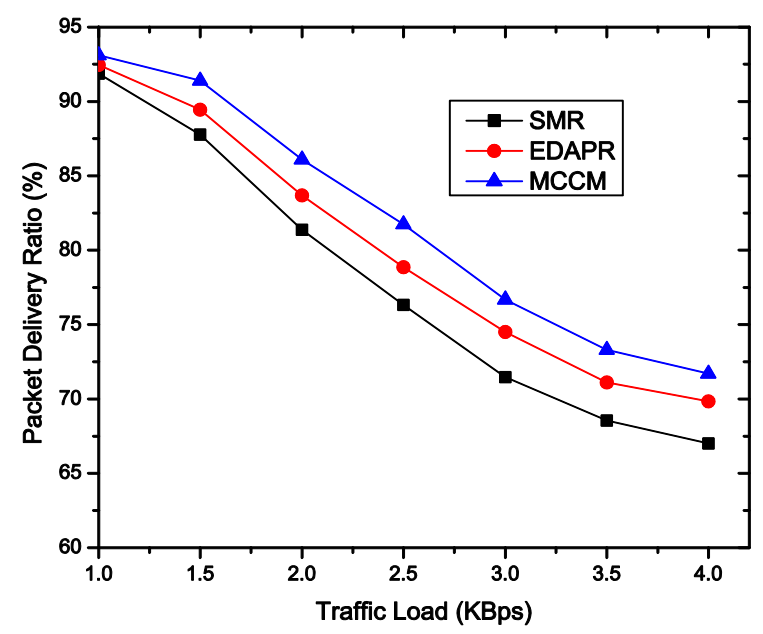

(a) Packet Delivery Ratio

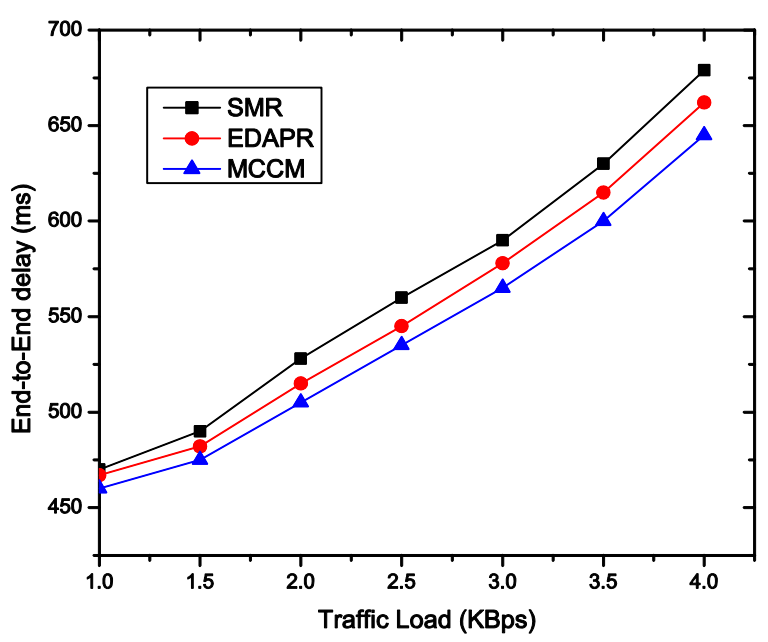

(b) Average end-to-end Delay

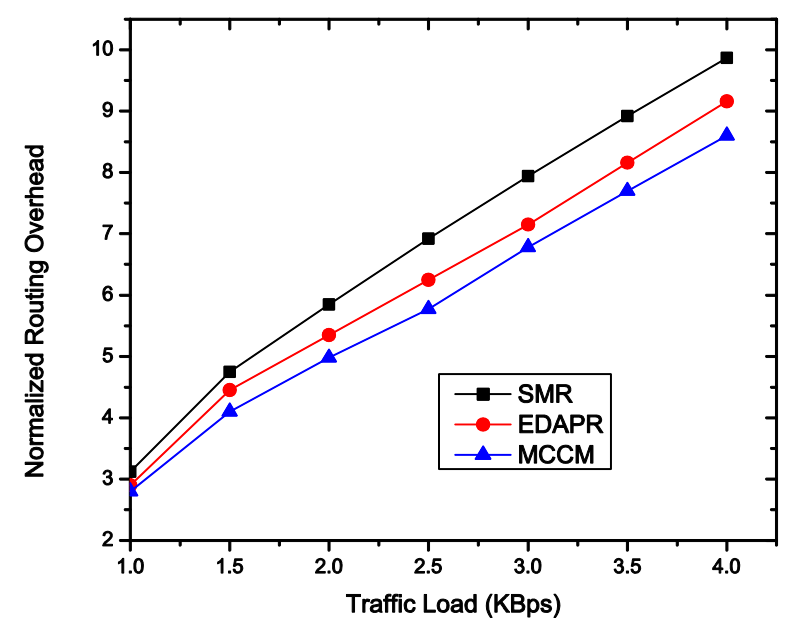

(c) Normalized Routing Overhead

Fig. 6. Performance comparisons for different traffic loads, mobility speed $=2 \mathrm{~m} / \mathrm{s}$.
Fig. 6(c) shows the normalized routing overhead of the studied protocols. In SMR, maintaining multiple routes creates lot of control packets generation since it has to keep information all of this routes. Besides, flooding of control packets during route discovery process for multiple routes causes huge flooding of RREQ and RREP packets. Thus, the normalized routing overhead for each successfully delivered data packets is high. For EDAPR, does not need to discover multiple routes and provides less routing overhead than SMR. But keeping information about each non-congested nodes (NHN) need to exchange a lots of control packets. On the other hand, in MCCM intermediate nodes do not develop maintain backup routes or NHN node leading less number of control packets to be exchange during the whole simulation period. As a result, MCCM it outperforms than other protocols and experiences lowest routing overhead.

\subsection{Impact of varying route failure rates}

We evaluate the impact of varying route failure rates and compare the result of MCCM with the other studied protocol. We keep the packets generation rate constant at 4 packets per seconds (i.e., $2 \mathrm{KBps}$ ). We vary the route failure rates from 1 10 routes/sec. The graphs in Fig. 7(a) show that, the packet delivery ratio for the all protocols decrease drastically with the increasing route failure rates. This happens because, increasing route failure increases the number of packet drops. The graph also shows that SMR has the lowest packet delivery ratio since multiple route breaks up lead huge data packets to be dropped during the simulation. The EDAPR does not maintain the energy efficient route and thus has lower ratio than proposed MCCM. Since the MCCM can find an energy efficient path during the route discovery process, it can deliver more data packets. The longer lifetime of MCCM provides high packet delivery rate than other protocols. Besides, energy efficient route selection methodology helps MCCM to deliver the data packets in an efficient manner by using the network resources like battery energy.

As expected theoretically, the end-to-end packet delivery delay increases with the route failure rates for all the protocols, as shown in Fig. 7(b). SMR experiences the longest end-to-end packet delivery delay since it drops large number of data packets during the link failure. Because our proposed capable of finding an energy efficient route, the longest lifetime causes the data packets delivery process effectively. As a result it experience minimum end-to-end delay than the others protocols.

Fig. 7(c) shows the normalized routing overhead and indicates that it will increase with the increasing route failures. SMR experience highest overhead. In SMR, the route failure rate leads to initiate fresh route discovery process to deliver the packets which increases the control packet generation rate. By selecting the most efficient path MCCM ensure the maximum use of the route before it become a broken route. Thus, MCCM has lowest routing overhead than the SMR and EDAPR. 


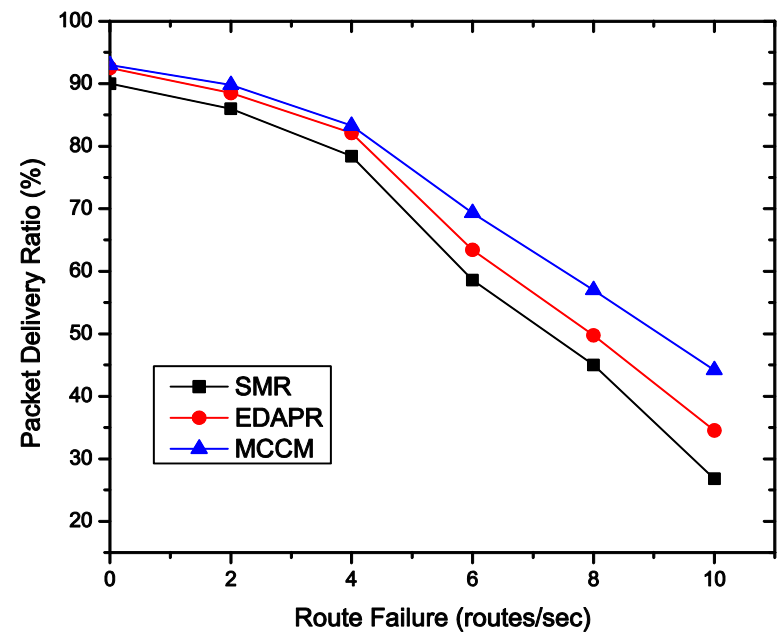

(a) Packet Delivery Ratio

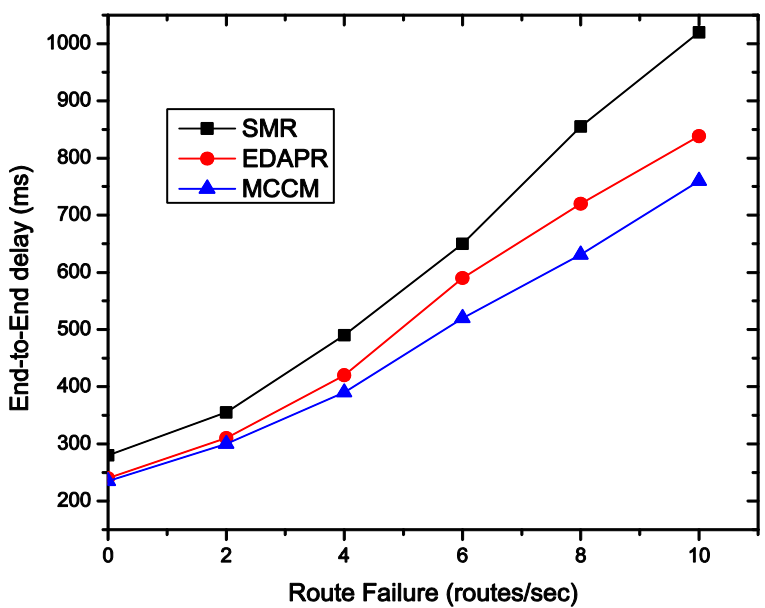

(b) Average end-to-end Delay

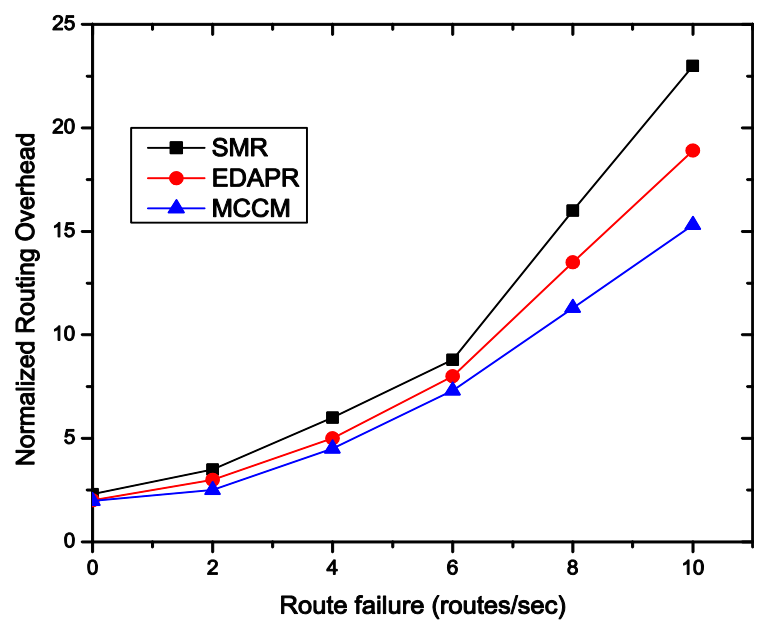

(c) Normalized Routing Overhead

Fig. 7. Performance comparisons for different route failure rates

\section{CONCLUSION}

In MANETs, congestion frequently leads to packets loss or delay in packets transmission. Our proposed
MCCM mechanism capable of developing an energy efficient path that ensures maximum use of network resources. The multilevel congestion detection and control mechanism of MCCM improves network performance significantly. The selective data delivery mechanism provides an effective way to mitigate congestion and ensures high data delivery rate, lower end-to-end delay and normalized routing overhead. Thus, MCCM outperforms the state-of-the art protocols and provide high throughput.

\section{ACKNOWLEDGEMENTS}

This work was supported jointly by the Networking Research Lab, Department of Information and Communication Technology, Mawlana Bhashani Science and Technology University and Green Networking Research Lab, Department of Computer Science and Engineering, University of Dhaka, Bangladesh. The authors are grateful for this support. Md. Manowarul Islam is the corresponding author of this paper.

\section{REFERENCES}

[1] Elizabeth $\mathrm{M}$ Royer and $\mathrm{Ck}$ Toh. A review of current routing protocols for ad hoc mobile wireless networks. IEEE Personal Communications, 6(2):46- 55, 1999.

[2] Charles E. Perkins and Elizabeth M. Royer. Ad-hoc ondemand distance vector routing. In proceeding of the 2 nd IEEE workshop on Mobile Computing Systems and Applications, pages 90-100, 1997.

[3] Kyungtae Woo, Chansu Yu, Dongman Lee, Hee Yong Youn, and Ben Lee. Non-blocking, localized routing algorithm for balanced energy consumption in mobile ad hoc networks. In Proceedings of the Ninth International Symposium in Modeling, Analysis and Simulation of Computer and Telecommunication Systems, MASCOTS '01, pages 117-, Washington, DC, USA, 2001. IEEE Computer Society.

[4] Laura Marie Feeney. An energy consumption model for performance analysis of routing protocols for mobile ad hoc networks. Mob. Netw. Appl., 6(3):239-249, June 2001.

[5] By Marco Fotino and Floriano De Rango. Energy issues and energy aware routing in wireless ad hoc networks. Mobile Ad-Hoc Networks: Protocol Design, 2011.

[6] Christian Lochert, Bj"orn Scheuermann, and Martin Mauve. A survey on congestion control for mobile ad hoc networks: Research articles. Wirel. Commun. Mob. Comput., 7(5):655-676, June 2007.

[7] Laxmi Shrivastava, G.S.Tomar, and S. Bhadauria. A survey on congestion adaptive routing protocols for mobile ad-hoc networks. International Journal of Computer Theory and Engineering, 2(5):189- 196, April 2011.

[8] S. Santhosh baboo and B.Narasimhan. A hop-byhop congestion-aware routing protocol for heterogeneous mobile ad-hoc networks. International Journal of Computer Science and Information Security, 3(1), July 2009.

[9] Chieh yih Wan and Shane B. Eisenman. Coda: Congestion detection and avoidance in sensor networks. In Proceedings of ACM SenSys, pages 266-279. ACM Press, Nov. 2003. 
[10] Sung ju Lee and Mario Gerla. Split multipath routing with maximally disjoint paths in ad hoc networks, In Proceedings of IEEE International Conference on Communications, pages 3201-3205, Aug. 2002.

[11] Sally Floyd and Van Jacobson. Random early detection gateways for congestion avoidance. Aug, 1993

[12] Roy Leung, Roy Leung Jilei, Edmond Poon, Ah lot Charles Chan, and Baochun Li. Mp-dsr: A qosaware multipath dynamic source routing protocol for wireless ad-hoc networks. In IEEE LCNO1, pages 132-141, 2001.

[13] A Valarmathi and R M Chandrasekaran. Congestion aware and adaptive dynamic source routing algorithm with loadbalancing in manets. International Journal, 8(5):69, 2010.

[14] A. Valarmathi and RM. Chandrasekaran. Article:congestion aware and adaptive dynamic source routing algorithm with load-balancing in manets. International Journal of Computer Applications, 8(5):1-4, October 2010. Published By Foundation of Computer Science.

[15] Juan Hernandez-Serrano, Josep Pegueroles, and Miguel Soriano. Energy efficiency of load balancing in manet routing protocols. In Proceedings of the Sixth International Conference on Software Engineering, Artificial Intelligence, Networking and Parallel/Distributed Computing and First ACIS International Workshop on Self-Assembling Wireless Networks, SNPD-SAWN '05, pages 476-483, Washington, DC, USA, 2005. IEEE Computer Society.

[16] T. Senthil Kumaran and V. Sankaranarayanan. Early detection congestion and self-cure routing in manet. In Proceedings of Springer LNCS computer and information science, volume 142, pages 562-7. Conference Publications, 2011.

[17] T. Senthil Kumaran and V. Sankaranarayanan. Early detection congestion and control routing in manet. In Proceedings of the seventh IEEE and IFIP international conference on wireless and optical communications networks (WOCN 2010), pages 1-5. Conference Publications, Sept 2010.

[18] T. Senthil Kumaran and V. Sankaranarayanan. Early congestion detection and adaptive routing in manet. Egyptian Informatics Journal, 12(3):165 - 175, 2011.

[19] The Network Simulator NS-2. http://www.isi.edu/nsnam/ns/.

\section{Authors' Profiles}

Md. Manowarul Islam received the B.Sc. and M.S. degree in Computer Science and Engineering from the University of Dhaka, Bangladesh, in the year 2008 and 2010, respectively. Currently, he is serving in the department of Information and Communication Technology, Mawlana Bhashani Science and Technology University as a lecturer. His research interests include the area of modeling, analysis and optimization of protocols, congestion control for mobile ad hoc network, Wireless Sensor networks, Cognitive radio network and underwater sensor network etc.

Dr. Md. Abdur Razzaque received his B.S. degree in Applied Physics and Electronics and M.S. degree in Computer Science from the University of Dhaka, Bangladesh in 1997 and 1999, respectively. He obtained his $\mathrm{PhD}$ degree in Wireless Networking from the Department of the Computer Engineering, School of Electronics and Information, Kyung Hee University, South Korea in August, 2009. He was a research professor, College of Electronics and Information, Kyung Hee University,
South Korea during 2010-2011. He is now working as an Associate Professor in the Department of Computer Science and Engineering, University of Dhaka, Bangladesh. He is the group leader of Green Networking Research Group (http://gnr.cse.univdhaka.edu) of the same department. He has been teaching a good number of courses related to Computer Networks, Wireless and Mobile Systems, Security, Information Technology Project Management, etc. to graduate and undergraduate students of reputed universities. He is also working as the principal investigators of some national and international research projects funded by Government of Bangladesh and Information Society Innovation Fund (ISIF) Asia. His research interest is in the area of modeling, analysis and optimization of wireless networking protocols and architectures, Wireless Sensor Networks, Body Sensor Networks, Cooperative Communications, Sensor Data Clouds, Cognitive Radio Networks, etc. He has published a good number of research papers in international conferences and journals. He is an editorial board member of International Journal of Network and Distributed Systems. He is an editorial board member of International Journal of Distributed Sensor Networks, TPC member of IEEE HPCC 2013, ICOIN 20102014, ADM 2014. He is a member of IEEE, IEEE Communications Society, IEEE Computer Society, Internet Society (ISOC), Pacific Telecommunications Council (PTC) and KIPS

Md. Ashraf Uddin received the B.Sc. and M.S. degree in Computer Science and Engineering from the University of Dhaka, Bangladesh, in the year 2008 and 2010, respectively. He worked as a faculty member in Department of Computer Science and Engineering, Bangladesh University of Business and Technology, Bangladesh. He also worked as a faculty member in Department of Computer Science and Engineering, City University, Dhaka, Bangladesh. Currently, he is serving in the department of Computer Science and Engineering, Mawlana Bhashani Science and Technology University, Tangail, Bangladesh as a lecturer. His research interests include the area of modeling, analysis and optimization of protocols and architectures for underwater sensor networks, artificial intelligent, etc.

A.K.M. Kamrul Islam, received the B.Sc. and M.S. degree in Computer Science and Engineering from the Jahangirnagar University, Bangladesh, in the year 2006 and 2007, respectively. Currently, he is serving in the department of Computer Science and Engineering, Mawlana Bhashani Science and Technology University, Tangail, Bangladesh as a lecturer. He has completed his Bachelor and Master degree from Jahangirnagar University. His main research interests include Cloud Computing, Artificial Neural Network, Network Security, Vehicular Ad hoc Network, Wireless Sensor Network etc.

How to cite this paper: Md. Manowarul Islam, Md. Abdur Razzaque, Md. Ashraf Uddin, A.K.M Kamrul Islam,"MCCM: Multilevel Congestion Avoidance and Control Mechanism for Mobile Ad Hoc Networks", International Journal of Information Technology and Computer Science(IJITCS), vol.6, no.6, pp.918, 2014. DOI: $10.5815 /$ ijitcs.2014.06.02 